\title{
Chris Heyde's Work on Rates of Convergence in the Central Limit Theorem
}

Peter Hall ${ }^{1}$

The best-known result on rates of convergence in the central limit theorem is undoubtedly that of A.C. Berry and C.-G. Esseen, which describes the rate in the case of finite third moments. In particular, if $X, X_{1}, X_{2}, \ldots$ are independent and identically distributed random variables for which

$$
E|X|^{3}<\infty
$$

if $E(X)=0$ and $E\left(X^{2}\right)=1$, and if we define

$$
S_{n}=\sum_{i=1}^{n} X_{i}
$$

then

$$
\sup _{-\infty<x<\infty}\left|P\left(S_{n} \leq n^{1 / 2} x\right)-\Phi(x)\right| \leq A E|X|^{3} n^{-1 / 2}
$$

where $\Phi$ is the standard normal distribution function and $A$ denotes an absolute constant.

The bound at (2) is the best possible, in the sense that for many distributions the convergence rate is exactly that given by (2). For example, Esseen also showed that if the distribution of $X$ is non-lattice, and if (1) holds, then

$$
P\left(S_{n} \leq n^{1 / 2} x\right)=\Phi(x)+n^{-1 / 2} \frac{1}{6} E\left(X^{3}\right)\left(1-x^{2}\right) \phi(x)+o\left(n^{-1 / 2}\right),
$$

uniformly in $x$ as $n \rightarrow \infty$, where $\phi=\Phi^{\prime}$ is the standard normal density function. However, the simplicity of (2) and (3) tends to obscure some of the more delicate, and therefore more interesting, properties of rates of convergence. Chris Heyde played a central role in revealing and exploring those important subtleties.

To access these issues we should mention first that the Berry-Esseen bound, at (2), is readily generalised to the case of moments lower than the third. In particular, if we replace condition (1) by the less stringent assumption that

$$
E|X|^{2+\delta}<\infty
$$

where $0<\delta \leq 1$, then (2) admits the following more general form:

$$
\sup _{-\infty<x<\infty}\left|P\left(S_{n} \leq n^{1 / 2} x\right)-\Phi(x)\right| \leq A_{\delta} E|X|^{2+\delta} n^{-\delta / 2},
$$

\footnotetext{
${ }^{1}$ Department of Mathematics and Statistics, The University of Melbourne, Melbourne, VIC 3130, Australia e-mail: p.hall@ms.unimelb.edu.au
} 


\section{Chris Heyde's Work on Rates of Convergence in the Central Limit Theorem}

where the constant $A_{\delta}$ depends only on $\delta$, not on the distribution of $X$ or on $n$. We know from expansions such as (3) that we cannot expect (5) to hold for $\delta>1$, and of course (5) is degenerate when $\delta=0$. However, when $0<\delta<1$ result (5) is rather conservative. In fact, in this case it can be shown that if (4) holds then

$$
\sup _{-\infty<x<\infty}\left|P\left(S_{n} \leq n^{1 / 2} x\right)-\Phi(x)\right|=o\left(n^{-\delta / 2}\right) .
$$

From some viewpoints it is perhaps disappointing that the inequality (5) is so conservative for $\delta<1$. Moment conditions such as (4) are very important to statistics, and the architecture of probability theory would have been simpler had there been an elementary, concise interpretation of (4) in terms of convergence rates. One of the attractions of the Berry-Esseen theorem is that it gives (in many cases) an exact convergence rate under an elementary moment assumption. However, as Einstein once remarked, "If you are out to describe the truth, leave elegance to the tailor." The conservatism evidenced by (6) begs the question of what the truth might be on this occasion; what rates of convergence are characterised by the moment assumption (4)?

Heyde gave a beautiful answer to this question, so exquisite and memorable that it will endure while ever moment conditions are discussed in connection with the central limit theorem. He showed in [M12] that if $0<\delta<1$ then (4) holds if and only if

$$
\sum_{n=1}^{\infty} n^{(\delta / 2)-1} \sup _{-\infty<x<\infty}\left|P\left(S_{n} \leq n^{1 / 2} x\right)-\Phi(x)\right|<\infty .
$$

Results of [M7] are related. In [M45], Heyde and Leslie derived analogous properties connecting finiteness of moments to approximations by Edgeworth series.

Condition (4) is rather uninformative if $\delta=0$. In that instance it tells us that $X$ has finite variance, and so the constants $n^{1 / 2}$ give an appropriate standardisation in the central limit theorem, but the amount of additional information it provides seems marginal, at least on the surface. Nevertheless, (7) is meaningful when $\delta=0$, and we are bound to ask what moment conditions are equivalent to that property, i.e. to:

$$
\sum_{n=1}^{\infty} n^{-1} \sup _{-\infty<x<\infty}\left|P\left(S_{n} \leq n^{1 / 2} x\right)-\Phi(x)\right|<\infty .
$$

In [M12] Heyde solved this problem, too, showing that (8) holds if and only if

$$
E\left\{X^{2} \log (1+|X|)\right\}<\infty .
$$

In [M60] Chris extended these results to rates of convergence in $L_{p}$ metrics, showing, among other results, that if $0<\delta<1$ then (4) is equivalent to

$$
\sum_{n=1}^{\infty} n^{(\delta / 2)-1}\left\|F_{n}-\Phi\right\|_{p}<\infty
$$

for $1 \leq p \leq \infty$, where $F_{n}(x)=P\left(S_{n} \leq n^{1 / 2} x\right)$ and, with $\Delta=F_{n}-\Phi$,

$$
\|\Delta\|_{p}= \begin{cases}\left\{\int_{-\infty<x<\infty}|\Delta(x)|^{p} d x\right\}^{1 / p} & \text { if } 1 \leq p<\infty, \\ \sup _{-\infty<x<\infty}|\Delta(x)| & \text { if } p=\infty .\end{cases}
$$

Moreover, [M60] shows that if $\delta=0$ then (9) and (10) are equivalent. Heyde and Nakata in [M106] obtained related results addressing the effects of choice of $p$ on convergence rates.

Theory for convergence rates in the central limit theorem is exceptionally delicate, and so it is unlikely that results such as these, striking though they may be, probe the very depths of the convergence-rate problem. Indeed, one is left wondering just what kinds of convergence rates are achievable under the condition of finite variance alone. In this circumstance, faster rates are sometimes possible 
if the sum $S_{n}$ is centred not at its expected value but at a truncated moment, or is rescaled using a truncated measure of variance. Examples of such location and scale constants include

$$
\mu_{n}=n E\left\{X I\left(|X| \leq n^{1 / 2}\right)\right\}, \sigma_{n}^{2}=n E\left\{x^{2} I\left(|X| \leq n^{1 / 2}\right)\right\} .
$$

In [M33] and [M49], Heyde proved that if centring and scaling constants are chosen to give the fastest possible convergence rate then the series at (8) converges under solely the assumption of finite variance. Indeed, if $X_{1}, X_{2}, \ldots$ come from a distribution in the domain of partial attraction of the normal distribution, then

$$
E\left(X^{2}\right)<\infty
$$

if and only if

$$
\sum_{n=1}^{\infty} n^{-1} \inf _{c>0,-\infty<d<\infty} \sup _{-\infty<x<\infty}\left|P\left(S_{n} \leq c x+d\right)-\Phi(x)\right|<\infty .
$$

This equivalence continues to hold if we fix $d=0$ in (13), and seek only the infimum over $c>0$. Moreover, the series in (13) converges if we take either $(c, d)=\left(\sigma_{n}, \mu_{n}\right)$ or $(c, d)=\left(\sigma_{n}, 0\right)$, for each $n$, where $\mu_{n}$ and $\sigma_{n}$ are given by (11). A further investigation of convergence rates shows that choosing $(c, d)=\left(\mu_{n}, \sigma_{n}\right)$ can give improved convergence rates, relative to $(c, d)=\left(0, \sigma_{n}\right)$, although the improvements are not expressible in terms of convergence of the series at (13).

The equivalence of (12) and (13) was proved independently by Egorov (1973), although under the slightly stronger condition that the distribution of $X$ is in the domain of attraction, not the domain of partial attraction, of the normal law. In [M28] Chris had previously derived a similar result for rates of convergence in the $L_{1}$ metric. Egorov (1973) extended this to $L_{p}$ metrics for $1 \leq p \leq 2$, and Rozovski1 (1978) treated the case $p \geq 1$. Ibragimov (1966), Osipov and Petrov (1967) and many others, working from the 1960s to the 1980s, developed a variety of related results.

This multiplicity of authors indicates the considerable interest attached to this line of research at the time. Heyde was competing with some of the strongest probabilists anywhere, and obtained definitive results. His work was fundamental to our understanding of the connections between moment conditions and convergence rates in the standard central limit theorem, in cases where that theorem is only barely valid; that is, where little more than finite variance is assumed.

\section{References}

[1] Egorov, V.A. (1973). The rate of convergence to the normal law that is equivalent to the existence of the second moment. (In Russian.) Teor. Verojatnost. i Primenen. 18, 180-185.

[2] Ibragimov, I.A. (1966). On the accuracy of approximation by the normal distribution of distribution functions of sums of independent random variables. (In Russian.) Teor. Verojatnost. i Primenen 11, 632-655.

[3] Osipov, L.V. and Petrov, V.V. (1967). On the estimation of the remainder term in the central limit theorem. (In Russian.) Teor. Verojatnost. i Primenen 12, 322-329.

[4] Rozovski1́, L.V. (1978). The accuracy of an estimate of the remainder term in the central limit theorem. (In Russian.) Teor. Veroyatnost. i Primenen. 23, 744-761. 